\title{
Daprodustat: First Approval
}

\author{
Sohita Dhillon ${ }^{1}$
}

Published online: 3 September 2020

(c) Springer Nature Switzerland AG 2020

\begin{abstract}
Daprodustat (DUVROQ) is a small molecule inhibitor of hypoxia-inducible factor prolyl hydroxylase (PHD) developed by GlaxoSmithKline for the treatment of anaemia in patients with chronic kidney disease (CKD). Inhibition of PHD prevents degradation of hypoxia-inducible factor (HIF), leading to the production of erythropoietin and subsequent induction of erythropoiesis. In June, daprodustat received its first approval in Japan for the treatment of renal anaemia. Clinical studies of daprodustat are underway in multiple countries worldwide. This article summarizes the milestones in the development of daprodustat leading to this first approval for the treatment of renal anaemia.
\end{abstract}

\section{Daprodustat (DUVROQ): Key points}

A small molecule PHD inhibitor is being developed by GlaxoSmithKline for the treatment of anaemia in patients with CKD

Received its first approval on 29 June 2020 in Japan Approved for the treatment renal anaemia

\section{Introduction}

Progressive chronic kidney disease (CKD) is associated with several serious complications, including anaemia, increased incidence of cardiovascular disease, hyperlipidaemia and metabolic bone disease [1]. Anaemia of CKD is largely the result of the diseased kidney being unable to adequately

Enhanced material for this AdisInsight Report can be found at https://doi.org/10.6084/m9.figshare.12768536.

This profile has been extracted and modified from the AdisInsight database. AdisInsight tracks drug development worldwide through the entire development process, from discovery, through preclinical and clinical studies to market launch and beyond.

Sohita Dhillon

dru@adis.com

1 Springer Nature, Private Bag 65901, Mairangi Bay, Auckland 0754, New Zealand respond to hypoxia and/or anaemia by inducing erythropoietin (EPO) production [2]. Other factors contributing to the development of anaemia include iron and vitamin deficiency, infection and inflammation [3]. Hypoxia-inducible factor (HIF) $1 \alpha$ and HIF $2 \alpha$ are heterodimeric transcription factors mediating the cellular response to hypoxia by altering gene expression in certain cell types. This results in the increased production of EPO in the kidney and liver, which in turn promotes an erythropoietic response and upregulation of iron transport [2-4]. HIF $\alpha$ levels are regulated via the action of a family of HIF-prolyl hydroxylases (PHDs) that are important for maintaining the balance between oxygen availability and HIF activity [5]. PHDs tag HIF $\alpha$ for proteasomal degradation, and inhibition of these hydroxylases simulates conditions of mild hypoxia, leading to an erythropoietic response [5]. The central role of PHDs as the enzymatic gatekeepers of the adaptive response to hypoxia makes them attractive therapeutic targets for the treatment of anaemia.

Daprodustat (DUVROQ) is a small molecule inhibitor of PHD developed by GlaxoSmithKline for the treatment of anaemia in patients with CKD. On 29 June 2020 [6], daprodustat received its first approval in Japan for the treatment of renal anaemia [7]. In adults with CKD who are not undergoing dialysis, the recommended initial dosage of daprodustat in patients untreated with an erythropoiesis-stimulating agent (ESA) is $2 \mathrm{mg}$ (for $\mathrm{Hb}$ levels $\geq 9.0 \mathrm{~g} / \mathrm{dL}$ ) or $4 \mathrm{mg}$ (for $\mathrm{Hb}$ levels $<9.0 \mathrm{~g} / \mathrm{dL}$ ) given orally once daily, and in adults switching from an ESA is $4 \mathrm{mg}$ given orally once daily. In dialysis-dependent patients, the recommended initial dosage of daprodustat is $4 \mathrm{mg}$ administered orally once daily irrespective of whether patients are receiving ESAs or switching 


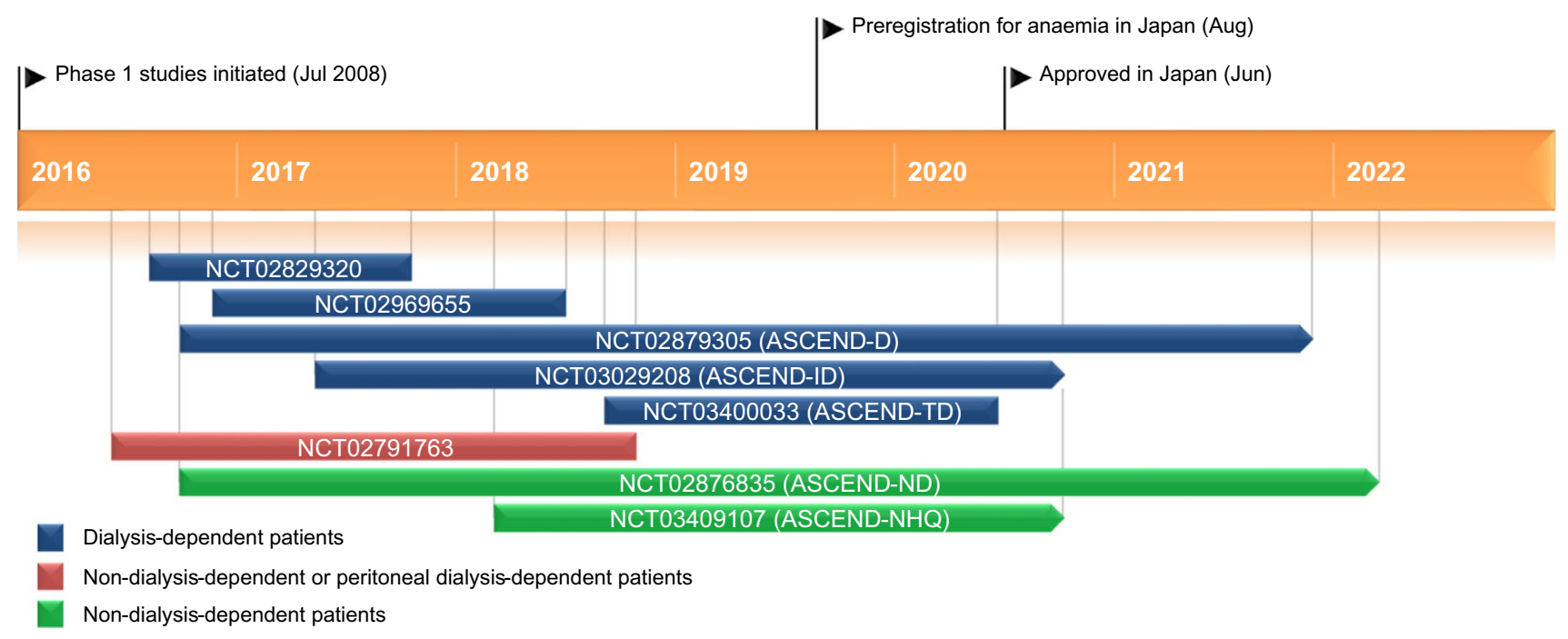

Key milestones in the development of daprodustat in the treatment of renal anaemia, focusing on phase 3 trials

from ESAs. Thereafter, daprodustat dose may be adjusted (maximum $24 \mathrm{mg}$ once daily) according to the severity of anaemia [7]. Clinical studies of daprodustat are underway in multiple countries worldwide. Development of daprodustat for diabetic foot ulcer, perioperative ischaemia, peripheral arterial disorders and tendon injuries has been discontinued.

\subsection{Company Agreements}

In November 2018 [8], GlaxoSmithKline (GSK) entered into an agreement with Kyowa Hakko Kirin for the commercialisation of daprodustat in Japan for use in patients with anaemia of CKD. Under the terms of the agreement, GSK is responsible for completion of the Japan clinical programme and regulatory submissions for marketing authorisation in Japan, while Kyowa Hakko Kirin is responsible for the distribution of daprodustat in Japan. Both the companies will jointly conduct launch activities, including engagement of healthcare professionals and commercial activities. Further financial details of the agreement were not disclosed [8]. The global programme for daprodustat outside of Japan is ongoing.

\section{Scientific Summary}

\subsection{Pharmacodynamics}

Daprodustat is a potent ( $\geq 1000$-fold selectivity) inhibitor of PHDs 1-3, which results in the stabilization of cellular HIF $1 \alpha$ and HIF $2 \alpha$, leading to the production of EPO and subsequent induction of erythropoiesis in in vivo preclinical studies [5]. In mice, a single oral dose of daprodustat increased EPO levels, with peak levels (11.2-fold higher than baseline) reached 12 hours after dosing. Increased production of EPO is believed to induce erythropoiesis, as demonstrated by significant ( $p<0.001$ vs. vehicle) increases in reticulocyte counts (211-673\% increase) and haemoglobin levels (12-17\% increase) in mice treated with oral daprodustat once daily for 8 days [5]. Daprodustat reduced mean ferritin levels, transferrin saturation and hepcidin levels and increased total iron binding capacity in non-dialysis-dependent patients, haemodialysis patients and peritoneal dialysis patients during $\leq 52$ weeks treatment in phase 3 studies [7].

At 75 and $500 \mathrm{mg}$ doses, daprodustat had no clinically significant effect on cardiac repolarization or QT interval (NCT02293148) [9].

\subsection{Pharmacokinetics}

The pharmacokinetic properties of oral daprodustat are based on data from healthy subjects and a population<smiles>O=C(O)CNC(=O)C1C(=O)N(C2CCCCC2)C(=O)N(C2CCCCC2)C1=O</smiles>

Chemical structure of daprodustat 
pharmacokinetic analysis based on data from non-dialysisdependent patients, haemodialysis patients and peritoneal dialysis patients with anaemia of CKD [7].

Daprodustat exhibited linear pharmacokinetics after single-dose administration over the dose range $10-100 \mathrm{mg}$. Following administration of a single oral $4 \mathrm{mg}$ dose of daprodustat, the median time to peak plasma concentration of daprodustat $\left(\mathrm{t}_{\max }\right)$ was reached in $1.75 \mathrm{~h}$ (fasting state) or 2.75 h (after a meal) (NCT03493386) [7, 10]. Daprodustat exposure was slightly lower when the drug was administered after a standard CKD meal, as indicated by a $9 \%$ decrease in the area under the concentration-time curve (AUC) from 0 to infinity and an $11 \%$ decrease in the peak plasma concentration $\left(\mathrm{C}_{\max }\right)(\mathrm{NCT} 03493386)[7,10]$.

Following multiple-dose administration, the median $\mathrm{t}_{\max }$ of daprodustat was $1-3.25 \mathrm{~h}$ in healthy subjects receiving daprodustat $15-100 \mathrm{mg}$ once daily, and $1-4 \mathrm{~h}$ in patients with anaemia of CKD receiving daprodustat $1-24 \mathrm{mg}$ once daily [7]. The absolute bioavailability of daprodustat after oral administration of $6 \mathrm{mg}$ of this drug was $65 \%$ and its volume of distribution after intravenous administration was 14.3 L. Daprodustat $(0.2-10 \mu \mathrm{g} / \mathrm{mL})$ was highly $(\approx 99 \%)$ protein bound to human plasma proteins (mainly albumin), according to in vitro data [7].

Daprodustat is largely metabolized by CYP2C8 and to a small extent by CYP3A4 in in vitro studies [7]. After oral administration of a radiolabelled dose of daprodustat, $40 \%$ of total radioactivity in the plasma was accounted for by the parent drug and $60 \%$ by metabolites. Oral daprodustat is primarily excreted in the faeces $(73.6 \%$ of a radiolabelled dose), with renal excretion a secondary route of elimination $(21.4 \%)$. The mean urinary excretion of oral daprodustat was $<0.05 \%$ of the dose [7]. The elimination halflife of oral daprodustat after a single $4 \mathrm{mg}$ dose in healthy subjects was $3.24 \mathrm{~h}$ (fasting state) or $3.22 \mathrm{~h}$ (after a meal) (NCT03493386) [7, 10].

Moderate [estimated glomerular filtration rate (eGFR) $30-59 \mathrm{~mL} / \mathrm{min} / 1.73 \mathrm{~m}^{2}$ ) or severe (eGFR $15-29 \mathrm{~mL} /$ $\min / 1.73 \mathrm{~m}^{2}$ ) renal impairment did not affect the pharmacokinetics of daprodustat to a clinically meaningful extent (NCT02293148 and NCT02243306) [7, 11]. The AUC values of all daprodustat metabolites assessed were higher in anaemic non-dialysis-dependent CKD stage 3/4 subjects (up to 2.84-fold) and in anaemic subjects on haemodialysis (up to 6.2-fold) than in subjects with normal renal function $\left(\mathrm{CL}_{\mathrm{CR}} \geq 90 \mathrm{~mL} / \mathrm{min} / 1.73 \mathrm{~m}^{2}\right)$ [7, 11]. Mild (Child-Pugh A) or moderate (Child-Pugh B) hepatic impairment also did not affect the pharmacokinetics of daprodustat $[7,12]$. As daprodustat is metabolized by CYP2C8, coadministration with CYP2C8 inhibitors may increase the plasma concentration of daprodustat (NCT01376232 and NCT02371603) [7, $13,14]$, and coadministration with CYP2C8 inducers may reduce the plasma concentration of daprodustat [7].

\section{Features and properties of daprodustat}

\begin{tabular}{|c|c|}
\hline Alternative names & 1278863; DUVROQ ${ }^{\text {a }}$; GSK-1278863; GSK-1278863A \\
\hline Class & Anti-ischaemics; antianaemics; pyrimidines; skin disorder therapies; small molecules \\
\hline Mechanism of action & $\begin{array}{l}\text { Inhibits of prolyl hydroxylases, thereby preventing the degradation of hypoxia-inducible factor, } \\
\text { leading to the production of erythropoietin and subsequent induction of erythropoiesis }\end{array}$ \\
\hline Route of administration & Oral \\
\hline \multirow[t]{2}{*}{ Pharmacodynamics } & Increased erythropoietin levels, reticulocyte counts and haemoglobin levels in mice \\
\hline & $\begin{array}{l}\text { Reduced mean ferritin levels, transferrin saturation and hepcidin levels and increased the total iron } \\
\text { binding capacity in patients with anaemia of chronic kidney disease }\end{array}$ \\
\hline Pharmacokinetics & $\mathrm{T}_{\max } 1-4 \mathrm{~h}$, plasma protein binding $\approx 99 \%$, excreted largely in the faeces, $\mathrm{t}_{1 / 2} \approx 3 \mathrm{~h}$ \\
\hline \multicolumn{2}{|l|}{ Adverse effects } \\
\hline$<1 \%$ & Retinal haemorrhage, hypersensitivity (rash, dermatitis, urticaria) and high blood pressure \\
\hline Serious & Thromboembolism \\
\hline \multicolumn{2}{|l|}{ ATC codes } \\
\hline WHO ATC code & $\begin{array}{l}\text { B03 (antianemic preparations); C (cardiovascular system); C01 (cardiac therapy); D03 (prepara- } \\
\text { tions for treatment of wounds and ulcers); M09A-X (other drugs for disorders of the musculoskel- } \\
\text { etal system) }\end{array}$ \\
\hline EphMRA ATC code & $\begin{array}{l}\text { B3 (antianaemic preparations); C1 (cardiac therapy); C6A (other cardiovascular products); D3A } \\
\text { (wound healing agents); M5X (all other musculoskeletal products) }\end{array}$ \\
\hline Chemical name & $N$-[(1,3-Dicyclohexylhexahydro-2,4,6-trioxopyrimidin-5-yl)carbonyl]glycine \\
\hline
\end{tabular}

${ }^{\text {a }}$ Trademarks are owned by or licensed to the GSK group of companies 


\subsection{Therapeutic Trials}

\subsubsection{Phase 3 Studies}

Daprodustat achieved and maintained target $\mathrm{Hb}$ levels (10-12 g/dL) during 24 weeks' treatment in Japanese haemodialysis patients who were not receiving an ESA, according to results of an open-label, multicentre phase 3 study (NCT02829320) [15]. Patients $(n=28)$ aged $\geq 20$ years with Hb levels $\geq 8$ to $<10 \mathrm{~g} / \mathrm{dL}$ and ferritin levels $>100 \mathrm{ng} / \mathrm{mL}$ received dosages of daprtodustat (determined as per pre-defined treatment algorithm) for 24 weeks. The mean change in $\mathrm{Hb}$ from baseline to week 4 (coprimary endpoint) was $0.79 \mathrm{~g} / \mathrm{dL}$ (mean $\mathrm{Hb}$ level increased from $9.10 \mathrm{~g} / \mathrm{dL}$ at baseline to $9.90 \mathrm{~g} / \mathrm{dL}$ at week 4$)$. The majority $(86 \%)$ of patients experienced an $\mathrm{Hb}$ increase of $>0$ to $2.0 \mathrm{~g} / \mathrm{dL}$, with $46 \%$ experiencing an increase of $>0$ to $1.0 \mathrm{~g} / \mathrm{dL}$ and $39 \%$ an increase of $>1.0$ to $2.0 \mathrm{~g} / \mathrm{dL}$ (coprimary endpoint). The target $\mathrm{Hb}$ level was achieved by week 8 and maintained within the target range over the 24-week period [15].

Daprodustat was noninferior to darbepoetin alfa in maintaining target $\mathrm{Hb}$ levels (10-12 g/dL) in Japanese haemodialysis patients with anaemia of CKD who were treated with ESAs, according to results of a randomized, double-blind, multicentre, phase 3 study (NCT02969655) [16]. Eligible patients were randomized to receive titration algorithm-determined dosages of daprodustat $(n=136)$ or darbepoetin alfa $(n=135)$ for 52 weeks. Mean Hb level at baseline was $10.94 \mathrm{~g} / \mathrm{dL}$ in the daprodustat group and $10.82 \mathrm{~g} / \mathrm{dL}$ in the darbepoetin group. During weeks 40-52 of treatment, the mean $\mathrm{Hb}$ level in patients receiving daprodustat was noninferior to that in patients receiving darbepoetin alfa (10.89 vs. $10.83 \mathrm{~g} / \mathrm{dL}$; primary endpoint), as the lower limit of $95 \%$ confidence interval was greater than the pre-specified noninferiority margin of -1.0 $\mathrm{g} / \mathrm{dL}$ (between-group difference $0.06 \mathrm{~g} / \mathrm{dL} ; 95 \% \mathrm{CI}-0.11$ to 0.23$)$. The majority of patients receiving daprodustat $(88 \%)$ or darbepoetin alfa $(90 \%)$ had $\mathrm{Hb}$ levels within the target range over weeks 40-52 of therapy [16].

Daprodustat was noninferior to epoetin beta pegol (an ESA) for the treatment of anaemia in non-dialysis-dependent Japanese patients with CKD (stages 3, 4 or 5; CKD3/4/5), according to results of a 52-week, open-label, multicentre study (NCT02791763) [7]. Patients (both ESA users and non-users) received the titration algorithm-determined dosages of daprodustat $(n=108)$ or epoetin beta pegol $(n=109)$ for 52 weeks. Following treatment with daprodustat or epoetin beta pegol (doses adjusted to achieve/ maintain haemoglobin levels of $10-13 \mathrm{~g} / \mathrm{dL}$ ), the mean haemoglobin level at the time of primary efficacy analysis (weeks 40-52) was noninferior between patients receiving daprodustat and those receiving epoetin beta pegol (11.97 vs. $11.86 \mathrm{~g} / \mathrm{dL}$; primary endpoint). The lower margin of the $95 \%$ CIs of the between-group difference $(0.10 ; 95 \%$ $\mathrm{CI}-0.07$ to 0.28 ) was above the non-inferiority margin of $-1.0 \mathrm{~g} / \mathrm{dL}$, indicating the noninferiority of the two treatment groups. The study also assessed the efficacy of daprodustat (titration algorithm-determined dosage) in treating anaemia in 56 patients on peritoneal dialysis. At the time of primary analysis (weeks 40-52), patients receiving daprodustat had a mean $\mathrm{Hb}$ level of $12.09 \mathrm{~g} / \mathrm{dL}$, which was within the target $\mathrm{Hb}$ level of 11-13 g/dL [7].

\subsubsection{Phase 2 Studies}

Daprodustat dose-dependently increased $\mathrm{Hb}$ levels over the first 4 weeks and maintained $\mathrm{Hb}$ target levels (10-11.5 g/dL) over 24 weeks of treatment in haemodialysis patients with stable Hb levels (9-11.5 g/dL) who were previously receiving a stable dose of recombinant human erythropoietin (rhEPO), according to a 24-week, randomized, open-label multicentre, global phase 2b study (NCT01977482) [17]. Patients were stratified by region (Japan vs. non-Japan) and prior rhEPO dose and randomized to receive daprodustat at starting doses of $4-12 \mathrm{mg}$ once daily $(n=177)$ or placebo $(n=39)$ for 4 weeks, with doses adjusted thereafter to achieve and maintain target $\mathrm{Hb}$ levels. The mean $\mathrm{Hb}$ level changed from baseline to week 4 (primary endpoint) in a dose-dependent manner in patients receiving daprodustat (mean change from baseline -0.29 to $0.69 \mathrm{~g} / \mathrm{dL}$ with daprodustat vs. -0.72 $\mathrm{g} / \mathrm{dL}$ ), with changes evident from week 2 [17].

Another 24-week, randomized, open-label, multicentre, phase 2b study (NCT01977573) showed that daprodustat effectively achieved/maintained target $\mathrm{Hb}$ levels over 24 weeks in non-dialysis-dependent CKD-3/4/5 patients with anaemia who were rhEPO naïve or who had switched from existing rhEPO therapy [18]. Patients who were rhEPO naïve $(n=180)$ were randomized 3:1 to receive daprodustat (1, 2 or $4 \mathrm{mg}$ based on baseline $\mathrm{Hb}$ ) once daily or control (rhEPO per standard of care), and rhEPO-users $(n=72)$ were randomized $1: 1$ to daprodustat $2 \mathrm{mg}$ once daily or control (rhEPO per standard of care) for 4 weeks, thereafter daprodustat doses could be adjusted to achieve and maintain target Hb levels (9-10.5 g/dL in Cohort 1 and 10-11.5 $\mathrm{g} / \mathrm{dL}$ in Cohort 2). At week 24, the mean Hb levels (primary endpoint) in the daprodustat group were $10.2 \mathrm{~g} / \mathrm{dL}$ (Cohort 1) and $10.9 \mathrm{~g} / \mathrm{dL}$ (Cohort 2) and in the control group were $10.7 \mathrm{~g} / \mathrm{dL}$ (Cohort 1) and $11.0 \mathrm{~g} / \mathrm{dL}$ (Cohort 2). Between weeks 12 and 24, target $\mathrm{Hb}$ levels were maintained within the target range in a median of $82 \%$ and $66 \%$ of patients in the daprodustat and control groups, respectively [18].

A 4-week, randomized, single-blind, multicentre, phase 2a study (NCT01047397) in anaemic non-dialysis-dependent patients with CKD-3/4/5 $(n=70)$ and anaemic haemodialysis patients with CKD stage 5D (CKD-5D; $n=37)$ 
determined that daprodustat induced a robust response (i.e. rate of increase and the absolute level of $\mathrm{Hb}$ ) and daprodustat doses of $<10 \mathrm{mg}$ once daily were suitable for further study in long-term clinical trials [19]. Patients were ESA-naive with $\mathrm{Hb}$ levels $\leq 11.0 \mathrm{~g} / \mathrm{dL}$ or if ESA treatment was discontinued for $\geq 7$ days or equivalent to the interval between scheduled ESA doses [19]. A 4-week, randomized, placebo-controlled phase 2a study $(n=72$; NCT01587898) in non-dialysis-dependent patients with anaemia of CKD (baseline $\mathrm{Hb} 8.5-11.0 \mathrm{~g} / \mathrm{dL}$ ) and who were not receiving rhEPO showed that daprodustat $0.5-5 \mathrm{mg}$ once daily produced dose-dependent increases in $\mathrm{Hb}$ levels, with the highest dose resulting in a mean increase of $1 \mathrm{~g} / \mathrm{dL}$ [20].

A 4-week, randomized, double-blind, placebo-controlled phase 2 study ( $n=97$; NCT02019719) in Japanese haemodialysis patients who were not receiving ESA (baseline $\mathrm{Hb}$ level 8.5-10.5 g/dL) also showed that daprodustat 4-10 mg once daily induced a dose-dependent increase in $\mathrm{Hb}$ levels relative to placebo [21]. In a 4-week, randomized, rhEPO-controlled phase 2a study ( $n=82$; NCT01587924), daprodustat $5 \mathrm{mg}$ was found to maintain mean $\mathrm{Hb}$ levels in haemodialysis patients with anaemia of CKD (baseline $\mathrm{Hb}$ 9.5-12.0 g/dL) who had switched from rhEPO; mean $\mathrm{Hb}$ levels decreased in patients receiving lower daprodustat doses ( 0.5 or $2 \mathrm{mg}$ once daily) [20]. A 29-day, randomized, double-blinded, placebo-controlled phase 2 study $(n=103$; NCT02689206) determined that daprodustat $10-30 \mathrm{mg}$ administered three times weekly in haemodialysis patients who were switched from stable doses of rhEPO (baseline $\mathrm{Hb} 9-11.5 \mathrm{~g} / \mathrm{dL}$ ) also produced dose-dependent increases in $\mathrm{Hb}$ levels [22].

\section{Key clinical trials of daprodustat sponsored by GlaxoSmithKline}

\begin{tabular}{|c|c|c|c|c|c|}
\hline $\operatorname{Drug}(\mathrm{s})$ & Indication & Phase & Status & Location(s) & Identifier \\
\hline Daprodustat & CKD anaemia in HD pts & 3 & Completed & Japan & NCT02829320; 204716 \\
\hline Daprodustat, darbepoetin alfa & CKD anaemia in HD pts & 3 & Completed & Japan & NCT02969655; 201754 \\
\hline Daprodustat, epoetin beta pegol & CKD anaemia in ND or PD pts & 3 & Completed & Japan & NCT02791763; 201753 \\
\hline Daprodustat, epoetin alfa & CKD anaemia in HD pts & 3 & Completed & Multinational & $\begin{array}{l}\text { NCT03400033; 204837; } \\
\text { 2017-004372-56; } \\
\text { ASCEND-TD }\end{array}$ \\
\hline Daprodustat placebo & CKD anaemia in ND pts & 3 & Ongoing & Multinational & $\begin{array}{l}\text { NCT03409107; 205270; } \\
\text { 2017-002270-39; } \\
\text { ASCEND-NHQ }\end{array}$ \\
\hline Daprodustat, darbepoetin alfa & CKD anaemia in ID & 3 & Ongoing & Multinational & $\begin{array}{l}\text { NCT03029208; 201410; } \\
\text { 2016-000507-86; } \\
\text { ASCEND-ID }\end{array}$ \\
\hline Daprodustat, rhEPO & CKD anaemia in HD pts & 3 & Ongoing & Multinational & $\begin{array}{l}\text { NCT02879305; 200807; } \\
\text { 2016-000541-31; } \\
\text { ASCEND-D }\end{array}$ \\
\hline Daprodustat, darbepoetin alfa & $\mathrm{CKD}$ anaemia in ND pts & 3 & Recruiting & Multinational & $\begin{array}{l}\text { NCT02876835; 200808; } \\
\text { 2016-000542-65; } \\
\text { ASCEND-ND }\end{array}$ \\
\hline Daprodustat, placebo & CKD anaemia in ND and HD pts & 2 & Completed & Multinational & $\begin{array}{l}\text { NCT01047397; 112844; } \\
\text { PHI112844 }\end{array}$ \\
\hline Daprodustat, placebo & CKD anaemia in ND pts & 2 & Completed & $\begin{array}{l}\text { USA, Canada, } \\
\text { Germany }\end{array}$ & NCT01587898; 116581 \\
\hline Daprodustat, rhEPO & $\mathrm{CKD}$ anaemia in $\mathrm{HD}$ pts & 2 & Completed & Multinational & NCT01587924; 116582 \\
\hline Daprodustat, rhEPO & $\mathrm{CKD}$ anaemia in ND pts & 2 & Completed & Multinational & NCT01977573; 113747 \\
\hline Daprodustat, rhEPO & CKD anaemia in HD pts & 2 & Completed & Multinational & NCT01977482; 113633 \\
\hline Daprodustat, placebo & CKD anaemia in HD pts & 2 & Completed & Japan & NCT02019719; 116099 \\
\hline Daprodustat, placebo & CKD anaemia in HD pts & 2 & Completed & Multinational & $\begin{array}{l}\text { NCT02689206; 204836; } \\
\text { 2015-004790-32 }\end{array}$ \\
\hline Daprodustat, epoetin alfa & CKD anaemia in HD pts & 2 & Recruiting & USA & $\begin{array}{l}\text { NCT03029247; 205665; } \\
\text { ASCEND-BP }\end{array}$ \\
\hline Daprodustat, rhEPO & CKD anaemia in ND pts & 2 & $\begin{array}{l}\text { Recruiting } \\
\text { (suspended) }\end{array}$ & USA & $\begin{array}{l}\text { NCT03457701; 201771; } \\
\text { ASCEND-Fe }\end{array}$ \\
\hline
\end{tabular}

$H D$ haemodialysis-dependent, $I D$ incident dialysis, $L-N M M A$ L- $N$-monomethyl arginine acetate, $N D$ non-dialysis-dependent, $P D$ peritoneal dialysis-dependent, $p t s$ patients, $r h E P O$ recombinant human erythropoietin 


\subsection{Adverse Events}

Daprodustat was generally well tolerated in the phase 3 studies in haemodialysis patients with anaemia of CKD who were (NCT02969655) [16] or were not (NCT02829320) [15] treated with an ESA, as well as in non-dialysis-dependent and peritoneal dialysis patients with anaemia of CKD (NCT02791763) [7]. Adverse reactions reported with daprodustat include retinal haemorrhage, hypersensitivity (rash, dermatitis, urticaria) and high blood pressure (BP) [all $<1 \%$ in frequency]. Daprodustat has also been associated with serious thromboembolic AEs in $0.8 \%$ of patients, including AEs such as cerebral infarction ( $0.3 \%)$, pulmonary embolism $(0.3 \%)$, retinal vein occlusion $(0.3 \%)$, deep vein thrombosis $(0.3 \%)$, vascular access thrombosis (e.g. shunt occlusion; frequency unknown) [7].

In haemodialysis patients with anaemia of CKD who were not receiving an ESA (NCT02829320), treatment-emergent AEs were reported in $89 \%$ (25/28) of patients over 24 weeks' daprodustat therapy; AEs occurred in 82\% (9/11) of patients initiating dialysis and 94\% (16/17) of patients on maintenance dialysis [15]. The most common ( $\geq 2$ patients) treatment-emergent AEs with daprodustat were nasopharyngitis (32\% [9 of 28]), infected dermal cyst (7\% [2 of 28]), shunt occlusion (7\% [2 of 28]) and shunt stenosis (7\% [2 of 28]). The majority of AEs were of mild or moderate severity. No treatment-emergent $\mathrm{AE}$ resulted in discontinuation of therapy or withdrawal from the study. Two patients had treatmentrelated AEs, with a decrease in blood cholesterol reported in a patient on maintenance dialysis and erythema reported in a patient initiating dialysis. A total of four serious AEs occurred in three patients and included shunt occlusion (considered severe) in two patients and device dislocation (intraocular lens dislocation) in one patient; all AEs resolved, and none were considered treatment related. Of the ophthalmologic assessments undertaken for ocular AEs, only one ocular AE (retinal haemorrhage) was reported in the study [15].

In haemodialysis patients with anaemia of CKD who were treated with ESAs (NCT02969655), the most frequent (incidence $\geq 10 \%$ ) treatment-emergent AEs with daprodustat or darbepoetin alfa during 52 weeks' therapy were nasopharyngitis, diarrhoea, shunt stenosis, contusion and vomiting [16]. Most treatment-emergent AEs were of mild or moderate severity and no deaths were reported in the study. The incidence of ocular AEs (e.g. proliferative retinopathy, macular edema choroidal neovascularisation) was similar between the two treatment groups.

In non-dialysis-dependent patients with anaemia of CKD (NCT02791763), treatment-related AEs occurred in $6 \%(9 / 149)$ of patients receiving daprodustat, including increased $\mathrm{Hb}$ levels, increased BP, increase in eosinophil counts, hypertension, abdominal distention, epigastric pain, gastroesophageal reflux disease, retinal haemorrhage and cerebral infarction (each $<1 \% ; 1 / 149)$ [7]. In peritoneal dialysis patients with anaemia of CKD, treatment-related AEs were reported in 14\% (8/56) of patients, including nausea $(4 \% ; 2 / 56)$ and diarrhoea, cough, pulmonary embolism, pulmonary hypertension, retinal haemorrhage, liver function abnormality, decreased haemoglobin, acne-like dermatitis and deep vein thrombosis (each 2\%; 1/56) [7].

\subsection{Ongoing Clinical Trials}

Several phase 3 efficacy and safety trials of daprodustat are ongoing, including the randomized, double-blind ASCENDNHQ trial (NCT03409107) in $\approx 600$ non-dialysis-dependent patients with anaemia of CKD. The study is comparing the efficacy, safety and health-related quality of life in patients receiving daprodustat versus those receiving placebo. The primary endpoint of the study is the mean change from baseline to the evaluation period (weeks 24-28) in $\mathrm{Hb}$ levels and secondary endpoints include the proportion of patients with $\mathrm{a} \geq 1.0 \mathrm{~g} / \mathrm{dL}$ increase in $\mathrm{Hb}$ level, proportion of $\mathrm{Hb}$ responders and the mean change from baseline in the Short Form-36 (SF-36) questionnaire vitality domain score. Recruitment is underway in the randomized, open-label, multicentre, phase 3 ASCEND-ND trial (NCT02876835) that is comparing the efficacy and safety of daprodustat with that of darbepoetin alfa in an estimated 4500 non-dialysis-dependent patients with anaemia of CKD. The coprimary endpoints of the study are the time to first occurrence of adjudicated major adverse cardiovascular event (MACE) and the mean change from baseline in $\mathrm{Hb}$ levels over the evaluation period (weeks 28-52).

Also ongoing is the randomized, open-label, multicentre, phase 3 ASCEND-D trial (NCT02879305) in $\approx 2964$ dialysis patients with anaemia of CKD that is comparing the efficacy and safety of daprodustat with that of rhEPO following a switch from ESAs. The coprimary endpoints of the study are the time to first occurrence of MACE and the mean change from baseline in $\mathrm{Hb}$ levels over the evaluation period (weeks 28-52). In addition, the randomized, open-label, multicentre, phase 3 ASCEND-ID trial (NCT03029208) is evaluating the efficacy and safety of daprodustat versus that of rhEPO in $\approx 300$ patients with anaemia of CKD who are initiating dialysis. The primary endpoint of the study is the mean change from baseline over the evaluation period (weeks 28-52) in the $\mathrm{Hb}$ level and secondary endpoints include the monthly intravenous iron dose, change from baseline to week 52 in systolic BP (SBP), diastolic BP (DBP) and mean arterial BP (MAP). The phase 3 ASCEND-TD trial (NCT03400033) in haemodialysis patients with anaemia of CKD is also ongoing and is comparing the efficacy and safety of daprodustat with that of epoetin alfa. The primary endpoint is the mean change from baseline in $\mathrm{Hb}$ levels over the evaluation period (weeks 28-52). 
Recruitment is underway in the randomized, open-label, phase 2 ASCEND-BP trial (NCT03029247), which will assess the effect of daprodustat on BP in haemodialysis in an estimated 62 patients with anaemia of CKD who will switch from a stable dose of ESA. The primary endpoint of the study is the average of 6-h post dose SBP at day 57 and secondary endpoints include the average of 6-h post dose SBP, DBP and MAP at day 1. Recruitment was underway in the randomized, open-label, phase 2 ASCEND-Fe trial (NCT03457701), which was designed to compare the effect of daprodustat compared with rhEPO on oral iron absorption in non-dialysisdependent patients with anaemia of CKD; however, the study has been suspended due to the COVID-19 pandemic.

\section{Current Status}

On 29 June 2020 [6], daprodustat received its first approval in Japan for the treatment of renal anaemia [7].

Acknowledgements During the peer review process, the manufacturer of daprodustat was also offered an opportunity to review this article. Changes resulting from comments received were made on the basis of scientific and editorial merit.

\section{Declarations}

Funding The preparation of this review was not supported by any external funding.

Authorship and Conflict of interest S. Dhillon is a contracted employee of Adis International Ltd/Springer Nature, and declares no relevant conflicts of interest. All authors contributed to the review and are responsible for the article content.

Ethics approval, Consent to participate, Consent to publish, Availability of data and material, Code availability Not applicable.

\section{References}

1. Thomas R, Kanso A, Sedor JR. Chronic kidney disease and its complications. Prim Care. 2008;35(2):329-44, vii.

2. Sanghani NS, Haase VH. Hypoxia-inducible factor activators in renal anemia: current clinical experience. Adv Chronic Kidney Dis. 2019;26(4):253-66.

3. Bonomini M, Del Vecchio L, Sirolli V, et al. New treatment approaches for the anemia of CKD. Am J Kidney Dis. 2016;67(1):133-42.

4. Becker KA, Jones JJ. An emerging treatment alternative for anemia in chronic kidney disease patients: a review of daprodustat. Adv Ther. 2018;35(1):5-11.

5. Ariazi JL, Duffy KJ, Adams DF, et al. Discovery and preclinical characterization of gsk1278863 (daprodustat), a small molecule hypoxia inducible factor-prolyl hydroxylase inhibitor for anemia. J Pharmacol Exp Ther. 2017;363(3):336-47.

6. GlaxoSmithKline. GSK receives first regulatory approval for Duvroq (daprodustat) in Japan for patients with anaemia due to chronic kidney disease [media release]. 29 June 2020. http://www. gsk.com.
7. GlaxoSmithKline. Duvroq (Daprodustat): Japanese prescribing information. 2020. https://www.pmda.go.jp/PmdaSearch/ iyakuDetail/ResultDataSetPDF/340278_39990D4F1024_1_01. Accessed 14 Aug 2020.

8. GlaxoSmithKline, Kyowa Hakko K. GSK and Kyowa Hakko Kirin sign strategic commercialisation deal in Japan for daprodustat, a potential new oral treatment for anaemia associated with chronic kidney disease [media release]. 22 Nov 2018. https://www.gsk. com.

9. Caltabiano S, Collins J, Serbest G, et al. A randomized, placeboand positive-controlled, single-dose, crossover thorough QT/QTc study assessing the effect of daprodustat on cardiac repolarization in healthy subjects. Clin Pharmacol Drug Dev. 2017;6(6):627-40.

10. Yamada M, Osamura M, Ogura H, et al. A single-dose, openlabel, randomized, two-way crossover study in healthy Japanese participants to evaluate the bioequivalence and the food effect on the pharmacokinetics of daprodustat. Clin Pharmacol Drug Dev. 2020. https://doi.org/10.1002/cpdd.793.

11. Caltabiano S, Cizman B, Burns O, et al. Effect of renal function and dialysis modality on daprodustat and predominant metabolite exposure. Clin Kidney J. 2019;12(5):693-701.

12. Shaddinger B, Andrews S, Mahar K, et al. Pharmacokinetics of daprodustat and metabolites in subjects with normal and impaired hepatic function. Clin Pharmacol Ther. 2020;107(Suppl 1):S92.

13. Johnson BM, Stier BA, Caltabiano S. Effect of food and gemfibrozil on the pharmacokinetics of the novel prolyl hydroxylase inhibitor GSK1278863. Clin Pharmacol Drug Dev. 2014;3(2):109-17.

14. Caltabiano S, Mahar KM, Lister K, et al. The drug interaction potential of daprodustat when coadministered with pioglitazone, rosuvastatin, or trimethoprim in healthy subjects. Pharmacol Res Perspect. 2018;6(2):e00327.

15. Tsubakihara Y, Akizawa T, Nangaku M, et al. A 24-week anemia correction study of daprodustat in Japanese dialysis patients. Ther Apher Dial. 2020;24(2):108-14.

16. Akizawa T, Nangaku M, Yonekawa T, et al. Efficacy and safety of daprodustat compared with darbepoetin alfa in Japanese hemodialysis patients with anemia: a randomized, double-blind, phase 3 trial [abstract no. SaO036]. Nephrol Dial Transplant. 2019;34 (Suppl 1):a350.

17. Meadowcroft AM, Cizman B, Holdstock L, et al. Daprodustat for anemia: a 24-week, open-label, randomized controlled trial in participants on hemodialysis. Clin Kidney J. 2019;12(1):139-48.

18. Holdstock L, Cizman B, Meadowcroft AM, et al. Daprodustat for anemia: a 24-week, open-label, randomized controlled trial in participants with chronic kidney disease. Clin Kidney J. 2019;12(1):129-38.

19. Brigandi RA, Johnson B, Oei C, et al. A novel hypoxia-inducible factor-prolyl hydroxylase inhibitor (GSK1278863) for anemia in CKD: a 28-day, phase 2A randomized trial. Am J Kidney Dis. 2016;67(6):861-71.

20. Holdstock L, Meadowcroft AM, Maier R, et al. Four-week studies of oral hypoxia-inducible factor-prolyl hydroxylase inhibitor GSK1278863 for treatment of anemia. J Am Soc Nephrol. 2016;27(4):1234-44.

21. Akizawa T, Tsubakihara Y, Nangaku M, et al. Effects of daprodustat, a novel hypoxia-inducible factor prolyl hydroxylase inhibitor on anemia management in Japanese hemodialysis subjects. Am J Nephrol. 2017;45(2):127-35.

22. Bailey CK, Caltabiano S, Cobitz AR, et al. A randomized, 29-day, dose-ranging, efficacy and safety study of daprodustat, administered three times weekly in patients with anemia on hemodialysis. BMC Nephrol. 2019;20(1):372. 